\title{
Anticancer activity of repurposed hemostatic agent desmopressin on AVPR2-expressing human osteosarcoma
}

\author{
NATASHA TATIANA SOBOL ${ }^{1}$, LUISINA MARÍA SOLERNÓ ${ }^{1}$, BRADY BELTRÁN ${ }^{2}$, LILIANA VÁSQUEZ ${ }^{2}$, \\ GISELLE VANINA RIPOLL ${ }^{1,3}$, JUAN GARONA ${ }^{1,3^{*}}$ and DANIEL FERNANDO ALONSO ${ }^{1,3^{*}}$ \\ ${ }^{1}$ Center of Molecular and Translational Oncology, National University of Quilmes, Bernal B1876BXD, Buenos Aires, \\ Argentina; ${ }^{2}$ Precision Medicine Research Center, School of Medicine, University of San Martín de Porres, Lima 15024, \\ Perú; ${ }^{3}$ National Council of Scientific and Technical Research (CONICET), Buenos Aires C1425FQB, Argentina
}

Received July 8, 2020; Accepted December 3, 2020

DOI: $10.3892 /$ etm.2021.9998

\begin{abstract}
Osteosarcoma is the most prevalent primary bone malignancy. Due to its high aggressiveness, novel treatment strategies are urgently required to improve survival of patients with osteosarcoma, especially those with advanced disease. Desmopressin (dDAVP) is a widely used blood-saving agent that has been repurposed as an adjuvant agent for cancer management due to its antiangiogenic and antimetastatic properties. dDAVP acts as a selective agonist of the vasopressin membrane receptor type 2 (AVPR2) present in the microvascular endothelium and in some cancer cells, including breast, lung, colorectal and neuroendocrine tumor cells. Despite the fact that dDAVP has demonstrated its antitumor efficacy in a wide variety of tumor types, exploration of its potential anti-osteosarcoma activity has, to the best of our knowledge, not yet been conducted. Therefore, the aim of the present study was to evaluate the preclinical antitumor activity of dDAVP in osteosarcoma. Human MG-63 and U-2 OS osteosarcoma cell lines were used to assess in vitro and in vivo therapeutic effects of dDAVP. At low micromolar concentrations, dDAVP reduced AVPR2-expressing MG-63 cell growth in a concentration-dependent manner. In contrast, dDAVP exhibited no direct cytostatic effect on AVPR2-negative U-2 OS cells. As it would be expected for canonical AVPR2-activation, dDAVP raised intracellular cAMP levels in osteosarcoma cells, and coincubation with phosphodiesterase-inhibitor rolipram indicated synergistic antiproliferative activity. Cytostatic
\end{abstract}

Correspondence to: Dr Juan Garona or Dr Daniel Fernando Alonso, Center of Molecular and Translational Oncology, National University of Quilmes, R. Saenz Peña 352, Bernal B1876BXD, Buenos Aires, Argentina.

E-mail: juan.garona@unq.edu.ar

E-mail: dfalonso@unq.edu.ar

${ }^{*}$ Contributed equally

Key words: osteosarcoma, desmopressin, vasopressin membrane receptor type 2, cytostatic, drug repurposing effects were associated with increased apoptosis, reduced mitotic index and impairment of osteosarcoma cell chemotaxis, as evaluated by TUNEL-labeling, mitotic body count in DAPI-stained cultures and Transwell migration assays. Intravenous administration of dDAVP $(12 \mu \mathrm{g} / \mathrm{kg}$; three times per week) to athymic mice bearing rapidly growing MG-63 xenografts, was indicated to be capable of reducing tumor progression after a 4 -week treatment. No major alterations in animal weight, biochemical or hematological parameters were associated with dDAVP treatment, confirming its good tolerability and safety. Finally, AVPR 2 expression was detected by immunohistochemistry in $66 \%$ of all evaluated chemotherapy-naive human conventional osteosarcoma biopsies. Taking these findings into account, repurposed agent dDAVP may represent an interesting therapeutic tool for the management of osteosarcoma. Further preclinical exploration of dDAVP activity on orthotopic or metastatic osteosarcoma models are required.

\section{Introduction}

Osteosarcoma is a high-grade bone malignancy and represents the most common primary bone cancer in children and adolescents (1). Osteosarcoma tumors are characterized by highly proliferating and invasive spindle cells of mesenchymal origin, with immature osteoid matrix deposition. The current standard management strategy for this disease involves surgical resection of all clinically-detectable lesions and systemic pre- and postoperative multidrug chemotherapy. Depending on the clinical guidelines followed by each country or region, these chemotherapeutic regimens usually combine high-dose methotrexate with doxorubicin and cisplatin, or etoposide in addition to ifosfamide $(2,3)$. Despite incorporation of these multimodality therapy approaches, $30-40 \%$ of osteosarcoma patients still develop local recurrences or distant metastases, becoming an exceptionally challenging disease with elevated mortality rates (4). Alarmingly, while 5-year survival rates of up to $70 \%$ are achieved in high-income countries, prognosis in low- and middle-income countries (LMICs) is significantly poorer, with reported survival rates as low as $45 \%$ (5). In a context of economic inequalities and resource-constrained health-care systems, these disparities are linked to limited 
access to adequate treatment and high rates of diagnostic delays, leading to late stage cancer diagnosis and poor survival $(6,7)$. Considering that in the last few decades advances in osteosarcoma management and survival rates have reached a discouraging plateau, there is a critical need to identify novel therapeutic tools for osteosarcoma.

Drug repurposing in oncology comprises the identification and use of already-approved drugs with a non-oncology primary use for cancer treatment (8). This strategy arises as an attractive alternative to de novo drug development, offering considerable advantages, such as an already proven safety profile, which in consequence can facilitate clinical evaluation, low production costs and widespread availability $(9,10)$. Following this rationale, during the past years our group has explored and characterized the use of hemostatic drug desmopressin (1-deamino-8-D-arginin vasopressin; dDAVP) as an adjuvant agent in cancer treatment. dDAVP has been used as a blood-saving agent for nearly 50 years in patients with bleeding disorders or undergoing complex surgeries characterized by large blood loss (11). This compound is a 1st generation synthetic analog of the vasopressin hormone (AVP) and acts as a selective agonist for the vasopressin membrane receptor type 2 (AVPR2) expressed in microvascular endothelium (12) and several transformed tissues, including breast (13-15), prostate (16), lung (17) and colorectal cancer $(18,19)$. Agonistic stimulation of vascular AVPR2 is associated with a cyclic adenosine monophosphate (cAMP)-mediated acute release of several hemostatic factors into the bloodstream, including factor VIII, tissue-type plasminogen activator and von Willebrand factor (VWF). VWF is a multifaceted blood glycoprotein involved in different biological processes such as coagulation, vascular normalization, cancer cell apoptosis and metastatic resistance (20-22). Preclinical studies by our research group and others using breast, colorectal, and lung cancer models reported that activation of tumor AVPR2 by dDAVP triggers antiproliferative signaling mechanisms after cAMP/cAMP-dependent protein kinase (PKA) axis activation, resulting in cell cycle arrest and decreased cell survival and growth (14,16-18,23-25). Cytostatic activity showed to be AVPR2-dependent as dDAVP effects on malignant cells were completely reverted after AVPR2 phamacological blockade or siRNA-mediated receptor depletion was performed $(16,23,24)$. dDAVP administration to animals bearing human or murine carcinomas was also capable of reducing tumor vascularization, and such angiostatic effects were associated to proteolytic production of antiangiogenic protein angiostatin (ANG) (26). Interestingly, several preclinical animal studies, as well as veterinary trials, showed that administration of dDAVP at clinically relevant doses has a profound antimetastatic activity, impairing dissemination and progression of residual metastatic cells and showing benefits in terms of distant recurrences after tumor resection and overall survival (14,18,27-31). Taking into account its potential anticancer properties as well as its validated and safe hemostatic activity, a phase II dose-escalation trial was conducted exploring dDAVP use as an adjuvant therapy during breast cancer surgical excision (NCT01606072). A perioperative treatment using two $1 \mu \mathrm{g} / \mathrm{kg}$ i.v. doses was associated to reduced intraoperative bleeding, increased plasmatic VWF and a postsurgical drop of circulating tumor cells (15). In a second phase I/II clinical trial, safety and symptom control were assessed in rectal cancer patients with bleeding. As a result, after 4 days of treatment initiation, complete hemostatic control and reduction of tumor vascular perfusion were achieved in the vast majority of patients (32). Despite dDAVP deployed interesting and promising anticancer activity as a repurposed agent in a variety of experimental and clinical settings, its potential therapeutic benefits in osteosarcoma were never evaluated.

As previously mentioned, osteosarcoma is fatal for about one-third of the patients worldwide and effective therapeutic options are rather limited, especially for advanced or refractory disease. In order to improve patient's chances of survival there is an urgent need for developing novel cost-effective therapeutic strategies. In view of this scenario, our present work sought to study the anticancer activity of repurposed hemostatic drug dDAVP on human osteosarcoma experimental models. Apart from exploring direct in vitro cytostatic effects of the compound, our study aimed to explore AVPR2 target expression in osteosarcoma xenografts as well as human tissue samples, and to evaluate the impact of sustained dDAVP administration in osteosarcoma tumor progression.

\section{Materials and methods}

Drugs and compounds. dDAVP was obtained from Romikin S.A. following GMP standards. Peptide purity was 98-99\% as assessed by MS-HPLC. Peptide sequence: Mpr-Tyr-P he-Gln-Asn-Cys-Pro-DArg-Gly-NH2 (disulfide bridge between Mpr-Cys). In order to achieve work concentrations, dDAVP was diluted in phosphate buffered saline (PBS) as vehicle. PDE3/4 inhibitor rolipram (ROL) was purchased from Sigma-Aldrich; Merck KGaA. Control groups were treated with PBS as vehicle.

Tumor cell lines and culture conditions. MG-63 (ATCC ${ }^{\circledR}$ CRL-1427 ${ }^{\mathrm{TM}}$ ) and U-2OS (ATCC ${ }^{\circledR}$ HTB-96 ${ }^{\mathrm{TM}}$ ) human osteosarcoma cell lines were obtained from the American Type Culture Collection. Human breast MCF-7 (ATCC ${ }^{\circledR}$ HTB-22 ${ }^{\mathrm{TM}}$ ) and MDA-MB-231 (ATCC ${ }^{\circledR}$ HTB-26 $^{\text {TM }}$ ) or lung H-125 (ATCC ${ }^{\circledR}$ CRL-5801 ${ }^{\mathrm{TM}}$ ) cancer cell lines were used as controls for in vitro or in vivo AVPR2 expression and were also acquired from the American Type Culture Collection. Tumor cell lines were grown in Dulbecco's modified Eagle's medium (DMEM, Thermo Fisher Scientific Inc.) plus $10 \%$ fetal bovine serum (FBS, Natocor), $2 \mathrm{mM}$ glutamine and $80 \mu \mathrm{g} / \mathrm{ml}$ gentamycin in monolayer culture, at $37^{\circ} \mathrm{C}$ in a humidified atmosphere of $5 \% \mathrm{CO}_{2}$. Cells were harvested using a trypsin/EDTA solution (Thermo Fisher Scientific Inc.) diluted in PBS and routinely tested for mycoplasma.

\section{In vitro studies}

AVPR2 expression analysis in osteosarcoma cells. Total RNA of MG-63 and U-2 OS cells was purified from $1 \times 10^{6}$ cells using the EasyPure ${ }^{\circledR}$ (TransGen) RNA kit following the manufacturer's protocol. RNA was reverse-transcribed with SuperScript III first-Strand Synthesis System (Thermo Fisher Scientific Inc.). The following forward and reverse primers were used: For AVPR2 5'-CTGGCCAAGGACACTTCATC-3' and 5'-GAAGGCAGCTGAGCTTC-3', for hypoxanthine phosphoribosyl transferase 1 (HPRT1), 5'-AACGTCTTGCTC GAGATGTG-3' and 5'-GCTTTGATGTAATCCAGCAGG-3'. Quantitative real time polymerase chain reaction (RT-qPCR) 
was performed using the SYBR Green PCR Master Mix (Thermo Fisher Scientific Inc.) and StepOne Real-Time PCR System (Applied Biosystems; Thermo Fisher Scientific Inc.). Thermal cycling conditions were $48^{\circ} \mathrm{C}$ for $30 \mathrm{~min}, 95^{\circ} \mathrm{C}$ for $10 \mathrm{~min}, 40$ cycles of $95^{\circ} \mathrm{C}$ for $15 \mathrm{sec}$ followed by $60^{\circ} \mathrm{C}$ for $60 \mathrm{sec}$. Each sample was analysed in triplicate and mean cycle threshold values $(\mathrm{Ct})$ were used for further analysis. $\mathrm{Ct}$ values were normalized for HPRT1 expression levels. The AVPR2 expression in MCF-7 cells (positive control) was considered as ' 1 '. Relative quantification values were calculated as $2^{-\Delta \Delta C t}$.

Osteosarcoma cell growth and cytotoxicity studies. Antiproliferative activity against rapidly growing tumor cells was determined using 72-h cell growth assays and crystal violet staining. $2.5 \times 10^{3} \mathrm{MG}-63$ or U-2 OS cells in $200 \mu \mathrm{l}$ were plated in 96-well flat bottom plates in complete DMEM, allowed to attach, and $24 \mathrm{~h}$ later treated with $\operatorname{dDAVP}(0.1,1$ or $10 \mu \mathrm{M}$ ) or PBS for $72 \mathrm{~h}$. By the end of the experiment, cells were briefly fixed with methanol, stained with $0.5 \%$ crystal violet solution, washed and dried overnight. After addition of ethanol/acetic acid 3:1 solution on crystal violet-dyed cultures, absorbance was measured at $595 \mathrm{~nm}$. The optical density of PBS-treated control cells was taken as $100 \%$ of cell growth. Lack of direct cytotoxicity of dDAVP ( $1 \mu \mathrm{M}$ for $24 \mathrm{~h})$ on MG-63 semi-confluent monolayers was performed using the trypan blue dye exclusion assay as previously described (16).

Apoptosis. Apoptotic cells were detected by terminal deoxynucleotidyl transferase dUTP nick end labeling (TUNEL). First, 1x104 MG-63 cells were plated on 24-well flat bottom plates and allowed to attach overnight. After 24-h starvation, cells were treated for $48 \mathrm{~h}$ with dDAVP $(1 \mu \mathrm{M})$ in complete medium. After incubation, cells were fixed in $4 \%$ paraformaldehyde (PFA) in PBS. DNA strand breaks were labeled with digoxigenin-dUTP using DeadEnd ${ }^{\mathrm{TM}}$ Fluorometric TUNEL System according to the manufacturer's protocol (Promega Corp.). Slides were mounted with DAPI-containing mounting medium Vectashield ${ }^{\mathrm{TM}}$ (Vector Laboratories Inc.) and visualized using a Cytation-Gen5 Cell Imaging Multi-Mode Reader (Biotek Instruments Inc.). A total of 10 images per experimental condition were acquired for analysis. The percentage of apoptotic cells in MG-63 cultures was calculated as (number of TUNEL-positive cells/total cells) $x 100$. Apoptosis in MG-63 cultures was confirmed using cytotoxic agent cisplatin $(10 \mu \mathrm{M})$ as a positive control.

Mitotic index of osteosarcoma cells in vitro. Osteosarcoma cell cultures were analyzed for mitotic index calculation after 48-h treatment with dDAVP $(1 \mu \mathrm{M})$, using the same starvation, PFA-fixation and DAPI-staining protocol as in previously described apoptosis assay. Ten images per experimental group were used for quantification. Mitotic figures in all 4 basic stages of mitosis (prophase, metaphase, anaphase and telophase) were counted using a high-power field magnification (HPF) of 400-fold magnification (x400) and the percentage of mitotic cells was obtained after quantification of total cell number per HPF.

Assessment of cAMP levels. Activation of canonical adenylate cyclase/cAMP/PKA axis after dDAVP-mediated AVPR2 stimulation was preliminary assessed by measuring intracellular
cAMP levels in MG-63 cells. Semi-confluent osteosarcoma cultures were exposed to dDAVP $(1 \mu \mathrm{M})$ or PBS for $1 \mathrm{~h}$ and cAMP was quantified using a competitive enzymatic immunoassay cAMP EIA Kit (Cayman Chemical Co.) following the steps detailed by the supplier.

Osteosarcoma cell chemotaxis. Tumor cell migration was assessed using the Transwell ${ }^{\circledR}$ migration assay. After overnight starvation, $2.5 \times 10^{4} \mathrm{MG}-63$ cells were seeded into $8 \mu \mathrm{m}$ pore inserts in 24-well plates (JetBiofil) in serum-free growth medium. The lower chamber was filled with DMEM containing $10 \%$ FBS, which was used as a chemoattractant. After 24-h incubation with dDAVP $(0.1,1$ or $10 \mu \mathrm{M})$ cells in the upper surface of the membranes were gently removed with cotton swabs. Cells that migrated to the lower surface were fixed with PFA and stained with $0.5 \%$ crystal violet. Migrated cells in five randomly selected x400 HPF were counted and normalized to control.

\section{In vivo osteosarcoma xenograft progression}

Animals. Outbred athymic female N:NIH(S)-nu mice aged 8 weeks with a weight of approximately $20 \mathrm{~g}$, were purchased from the UNLP Animal Facility (National University of La Plata, School of Veterinary Sciences, Buenos Aires, Argentina), and housed at 5 mice per cage in our animal house facility at the National University of Quilmes. Food and water were provided ad libitum and general health status of the animals was monitored daily.

Tumor progression. To generate human osteosarcoma xenografts, a $150 \mu \mathrm{l}$-suspension containing $5 \times 10^{6} \mathrm{MG}-63$ cells in 1:0.5 volume ratio mixture of DMEM and Matrigel ${ }^{\circledR}$ (Becton, Dickinson and Company) was injected subcutaneously in athymic mice. Tumors were measured periodically with a calliper and tumor volume was calculated by the formula: $0.52 \times$ width $^{2} \mathrm{x}$ length. Animal weight and tumor growth rates were also assessed along the protocol. Four days after cell inoculation and once all tumors were detectable by palpation, administration of dDAVP began, using a $12 \mu \mathrm{g} / \mathrm{kg}$ i.v. dose, 3 times per week during 4 weeks. Animal dosing was determined by dose extrapolation using the 'dose by factor' method based on allometric scaling, following the United States Food and Drug Administration guidelines (33). A clinically validated dDAVP dose of $1 \mu \mathrm{g} / \mathrm{kg}$ i.v. was taken as the reference dose (15,30-32) and multiplied by 12.3 for human to mice conversion (33). Mice equivalent dose was rounded down from 12.3 to $12 \mu \mathrm{g} / \mathrm{kg}$ i.v. to facilitate drug preparation and administration. When lesions reached volumes $>300 \mathrm{~mm}^{3}$, and signs of tumor necrosis and skin infiltration were observed in the majority of tumors, animals were euthanized by cervical dislocation. Tumors were removed, weighted, fixed with $10 \%$ formalin and processed for hematoxylin and eosin (H\&E) or Masson trichrome staining in order to explore tumor histology.

Hematological and blood biochemical analysis. On the final day of the tumor progression protocol, after $1 \mathrm{~h}$ of dDAVP administration and before euthanasia, animals were anesthetized using intraperitoneal ketamine at $100 \mathrm{mg} / \mathrm{kg}$ in combination with $10 \mathrm{mg} / \mathrm{kg}$ xylazine, and EDTA-anticoagulated whole-blood samples from each of the experimental subjects were collected for further hematological and biochemical 

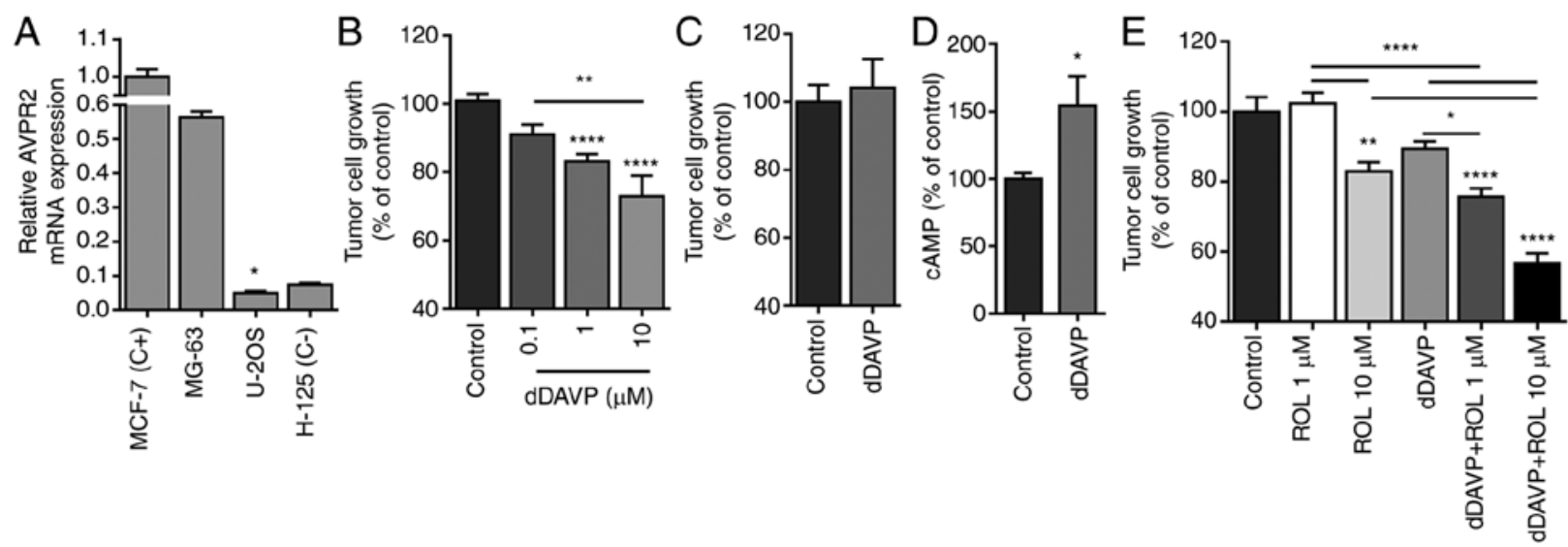

Figure 1. dDAVP reduces AVPR2-expressing osteosarcoma cell growth. (A) Assessment of targeted receptor AVPR2-expression in human osteosarcoma MG-63 and U-2 OS cell lines by reverse transcription-quantitative PCR. Human breast MCF-7 and lung H-125 cancer cells were used as positive and negative expression controls, respectively. (B) Inhibition of cell growth by dDAVP (0.1-10 $\mu \mathrm{M})$ on high-density AVPR2-positive MG-63 cell cultures. Cellular growth was assessed on exponentially growing osteosarcoma cell cultures and measured by crystal violet staining after a $72 \mathrm{~h}$-exposure to dDAVP. (C) Absence of cytostatic effect of dDAVP $(10 \mu \mathrm{M})$ on AVPR2-negative U-2 OS cells. (D) Intracellular cAMP levels in MG-63 cells after 1-h incubation with dDAVP (1 $\mu$ M). (E) Drug combination studies using of PDE3/4 inhibitor rolipram (ROL; 1 and $10 \mu \mathrm{M}$ ) in addition to dDAVP. Data are presented as mean \pm SEM. ${ }^{*} \mathrm{P}<0.05,{ }^{* *} \mathrm{P}<0.01$ and ${ }^{* * * * *} \mathrm{P}<0.0001$ vs. control or between different treatments as indicated by brackets. dDAVP, Desmopressin; AVPR2, vasopressin membrane receptor type 2 .

analysis. Hematocrite, red and white blood cell and platelet count, cholesterol, total protein, direct bilirubin, creatinine and albumin levels, as well as aspartate aminotransferase and alanine aminotransferase activity were assessed.

Immunohistochemical staining for AVPR2 in osteosarcoma xenografts or human samples. MG-63 osteosarcoma tumor samples were fixed in $10 \%$ formalin, embedded in paraffin, and tissue sections of $4 \mu \mathrm{m}$ were cut and placed on silane coated slides for anti-AVPR2 immunohistochemistry (IHC) staining. Sections were microwaved for antigen retrieval in $0.01 \mathrm{M}$ sodium citrate buffer (pH 6.0) during $20 \mathrm{~min}$, and later incubated with $3 \%$ hydrogen peroxide for $10 \mathrm{~min}$ to block endogenous peroxidase. Immunostaining was performed using an anti-AVPR2 primary polyclonal rabbit antibody (V5514; 1:100 dilution, Sigma-Aldrich; Merck KGaA) at room temperature for $1 \mathrm{~h}$. For visualizing immunoreactivity, a 15-min incubation using Super Sensitive $^{\mathrm{TM}}$ One-Step Polymer-HRP IHC Detection System (Bio-genex) was conducted. Finally, sections were counterstained with hematoxylin. MCF-7 and MDA-MB-231 breast cancer xenografts were generated in athymic nude mice by a subcutaneous injection of a $300 \mu \mathrm{l}$ suspension containing $5 \times 10^{6}$ cancer cells in a 1:1 volume ratio mixture of DMEM and Matrigel ${ }^{\circledR}$, as reported $(25,34,35)$. After 45 days of tumor progression, tumors were resected and processed as previously described, and were used as positive controls for in vivo AVPR2 expression. For expression analysis of targeted receptor in human tumor samples, formalin-fixed paraffin-embedded chemotherapy-naïve conventional osteosarcoma biopsy specimens were processed for IHC after diagnosis confirmation by the pathological anatomy service from the Edgardo Rebagliati Martins National Hospital. Additionally, histological characteristics of different osteosarcoma subtypes were assessed using hematoxylin and eosin (plus alcian blue) or Masson's trichrome (plus aniline blue) staining.

Statistical analysis. All statistical analyses were performed using the PRISM 6 (GraphPad Software Inc.) or Compusyn software (Combosyn Inc., New Jersey, United States).
To compare differences between 2 experimental groups, two-tailed Mann Whitney or t-tests were used for non-parametric or normal distribution of data, respectively. In case of more than 2 experimental groups, ANOVA analysis with Tukey's multiple comparisons post-test was used. Kruskal-Wallis analysis with Dunn's multiple comparisons post-test was used in case of non-parametric distribution of data. In drug combination studies synergy was determined when combination index (CI) was $<1$. The number of mitotic or apoptotic cells was expressed as percentage of positive cells/total number of cells counted in each specific condition ( $\pm 95 \%$ confidence limits), and analysed by the $\chi^{2}$ test. In tumor progression protocols, growth rates represent the slopes of the linear regressions of the tumor volumes over time. Differences were considered statistically significant at a level of $P<0.05$. Data were presented as mean \pm standard deviation (SD) or standard error of mean (SEM). Data were derived from at least two or three independent experiments, unless stated otherwise.

\section{Results}

In vitro treatment with dDAVP inhibits tumor cell growth in AVPR2-expressing osteosarcoma and is associated with elevated cAMP levels. First, AVPR2 expression was assessed in MG-63 and U-2 OS human osteosarcoma cells. RT-qPCR analysis showed comparable AVPR2 expression levels between MG-63 and MCF-7 cells (positive control). In contrast, AVPR2 gene expression in U-2 OS was almost undetectable (Fig. 1A). In accordance with these results, exposure of MG-63 semiconfluent monolayers to dDAVP $(0.1-10 \mu \mathrm{M})$, but not of AVPR2-negative U-2 OS cultures, resulted in a significant dose-dependent inhibitory effect over cell growth (Fig. 1B and C). Given that direct antiproliferative activity of dDAVP on tumor cells seems to be mediated by cAMP/PKA signalling-related pathways $(19,23)$, we assessed intracellular cAMP levels in MG-63 cultures after dDAVP treatment. As a result, a 54\% increase in cAMP intracellular concentration was observed after dDAVP treatment in osteosarcoma cells. 
Both in normal and malignant cells, elevated intracellular cAMP levels are rapidly restored by efflux and/or decomposition through different multidrug-resistance proteins and phosphodiesterases, respectively. We, therefore, evaluated the effect of phosphodiesterase inhibition using rolipram in combination with cAMP-elevating agent dDAVP. Osteosarcoma cells were incubated with dDAVP $(1 \mu \mathrm{M})$ plus effective $(10 \mu \mathrm{M})$ or sub-optimal $(1 \mu \mathrm{M})$ concentrations of rolipram, obtaining synergistic growth inhibitory effects in both tested drug combinations (Fig. 1E).

Cytostatic activity of dDAVP in osteosarcoma cells is associated with mitosis inhibition, increased apoptosis and antimigratory effects. In order to gain further insights into dDAVP anti-osteosarcoma effects, we preliminarily explored compound's capacity to modulate other key events in osteosarcoma tumor biology such as mitosis, apoptosis and migration. As shown in Fig. 2, a 48-h treatment with dDAVP reduced the percentage of MG-63 cells undergoing mitosis by nearly $70 \%$ in contrast to vehicle-treated cells. In equivalent experimental conditions, dDAVP at $1 \mu \mathrm{M}$ caused a 3.4-fold increase in the number of osteosarcoma apoptotic cells, as evaluated by DNA fragmentation labeling (Fig. 2A and B, respectively). Furthermore, Transwell chemotaxis assays demonstrated a significant decrease in the migratory capacity of osteosarcoma cells upon overnight incubation with tested compound (Fig. 2C). Lack of short-term cytotoxicity of dDAVP on osteosarcoma cells was confirmed using the trypan blue dye exclusion assay (Fig. 2D).

Sustained dDAVP treatment impairs osteosarcoma xenograft growth in athymic mice. In order to explore potential therapeutic activity on in vivo osteosarcoma growth, the effect of dDAVP treatment was evaluated in MG-63 xenograft-bearing athymic nude mice (Fig. 3). Sustained intravenous administration of dDAVP $(12 \mu \mathrm{g} / \mathrm{kg})$ caused a significant decrease in tumor volume over time (Fig. 3A), resulting in a tumor growth rate inhibition of $34 \%$ in comparison to control group (Fig. 3C). Impact of dDAVP treatment on final tumor burden was confirmed after observing a $25 \%$ reduction in resected tumor weight (Fig. 3D). Interestingly, despite observed significant differences regarding disease progression between groups, at the end of the protocol the majority of experimental subjects displayed necrotic and ulcerated lesions with erratic and jagged tumor growth patterns (Figs. 3B and S1A and B). dDAVP treatment had no impact on the general health status of treated animals as assessed by body weight (Fig. 3D), and blood biochemical and hematological studies. As observed in Table I, nearly all biochemical and hematological parameters remain unaltered after 1 month of drug administration. In consistency with the compound well characterized hemostatic action, a significant $13 \%$ drop in the mean platelet count was observed in animals receiving dDAVP.

AVPR2 expression in osteosarcoma xenografts and human biopsies. Histological assessment of rapidly growing MG-63 tumors revealed large necrotic foci with evident loose fibrous and reticular connective tissue deposition (Fig. 4A and B), and scattered areas of high cellularity showing typical spindle/fibroblastoid cell morphology (Fig. 4C). Large necrotic areas were found in all tumors, and could in part explain the
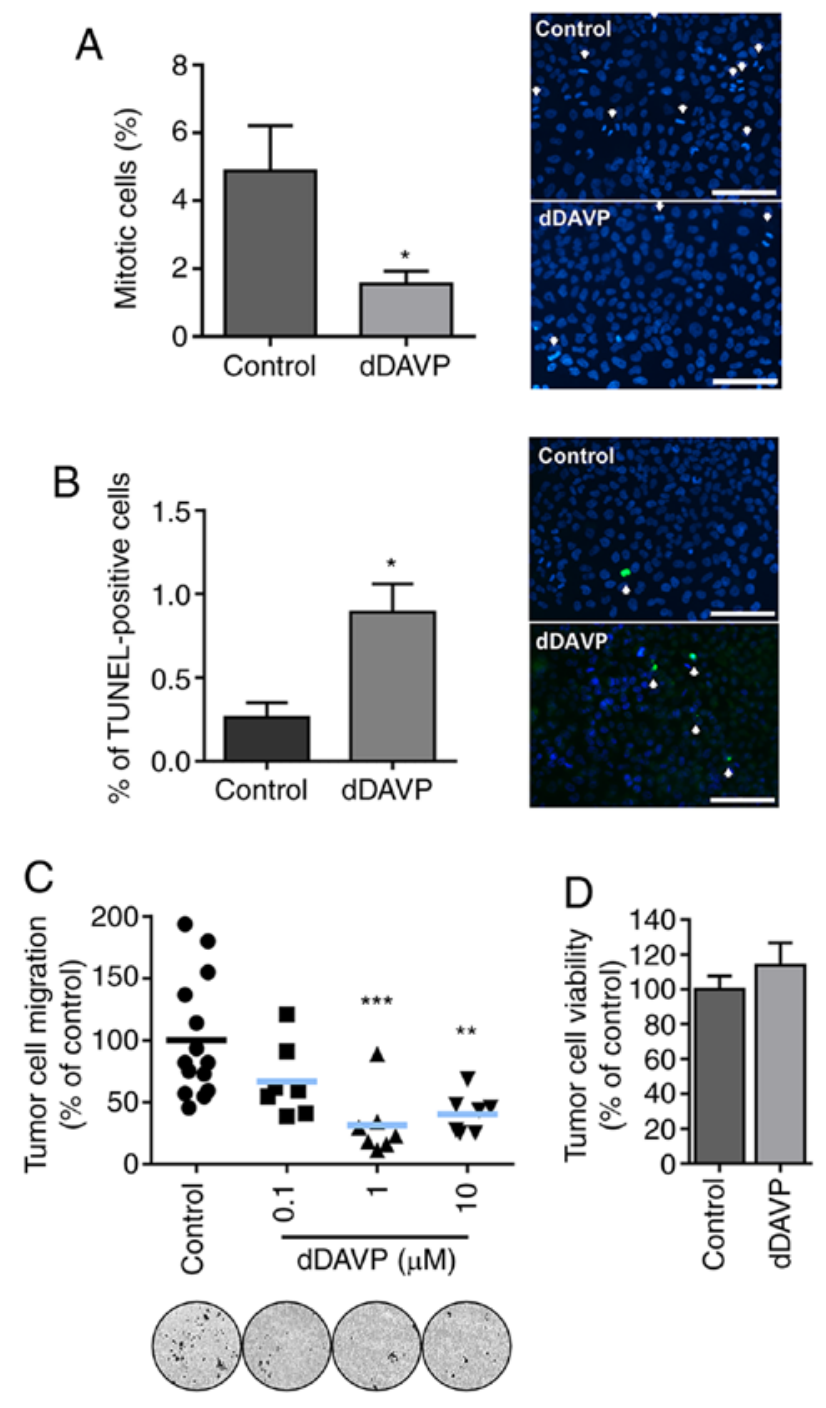

Figure 2. Direct cytostatic effects of dDAVP on osteosarcoma cells are associated to reduced mitosis, increased apoptosis and impaired tumor cell chemotaxis. (A) Reduction of cellular mitotic index on MG-63 cell cultures after a $48 \mathrm{~h}$ treatment with dDAVP $(1 \mu \mathrm{M})$. Quantification of mitosis (left) expressed as \% of mitotic cells/total cells. Magnification, x400. Scale bar, $100 \mu \mathrm{m}$. Representative images (right) of cell cultures under DAPI nuclear staining evidencing mitotic bodies. (B) Percentage (\%) of TUNEL-positive cells relative to total cell count after DAPI-stained nuclei quantification (left). Representative images of MG-63 apoptotic cells after $48 \mathrm{~h}$ exposure to dDAVP $(1 \mu \mathrm{M})$ (right). Magnification, $\mathrm{x} 400$. Scale bar, $100 \mu \mathrm{m}$. Arrows indicate mitotic bodies or TUNEL-positive apoptotic cells, respectively. (C) dDAVP impact on MG-63 cell migration after a $16 \mathrm{~h}$ treatment as assessed by Transwell migration assays. Representative images of migrated osteosarcoma cells on the bottom of the inserts (bottom, left to right, control and increasing concentrations of dDAVP). Magnification, x100. (D) 24 h viability assay using semiconfluent MG-63 cell monolayers exposed to dDAVP. Data presented as mean \pm SEM. ${ }^{*} \mathrm{P}<0.05,{ }^{* *} \mathrm{P}<0.01$ and ${ }^{* * *} \mathrm{P}<0.001$ vs. control group. Contingency $\chi^{2}$ test was used in (A) and (B). dDAVP, Desmopressin.

jagged growth patterns and irregular progression of tumors observed in all experimental subjects. In vivo confirmation of therapeutic target AVPR2 expression in MG-63 xenografts was conducted using IHC. Osteosarcoma tissue displayed a diffuse membrane and cytoplasmatic distribution of AVPR2 throughout the tumor, with variable staining intensity (Fig. 4D, E and F). Human MDA-MB-231 and MCF-7 breast cancer xenografts were used as positive controls for AVPR2 expression (Fig. 4H and I) (13). It is worth noting that, in 
Table I. Biochemical and hematological parameters assessed in EDTA-anticoagulated whole-blood from MG-63 xenograft-bearing athymic nude mice under dDAVP treatment.

Treatment group

Parameter

Control (saline, mean \pm SD)

$\operatorname{dDAVP}\left(1 \mu \mathrm{g} \mathrm{kg}^{-1}\right.$, mean $\left.\pm \mathrm{SD}\right)$

Weight $(\mathrm{g})^{\mathrm{a}}$

Hematocrit (\%)

$\operatorname{RBC}\left(10^{6} \mathrm{ml}^{-1}\right)$

WBC $\left(10^{3} \mathrm{ml}^{-1}\right)$

Platelets $\left(10^{6} \mathrm{ml}^{-1}\right)$

Cholesterol $\left(\mathrm{mg} \mathrm{dl}^{-1}\right)$

Total protein $\left(\mathrm{g} \mathrm{dl}^{-1}\right)$

Direct bilirrubin $\left(\mathrm{mg} \mathrm{dl}^{-1}\right)$

Creatinine $\left(\mathrm{mg} \mathrm{ml}^{-1}\right)$

AST/GOT $\left(\mathrm{IU} \mathrm{l}^{-1}\right)$

ALT (IU 1 ${ }^{-1}$ )

Plasmatic albumin $\left(\mathrm{g} \mathrm{dl}^{-1}\right)$

$$
\begin{gathered}
21.4 \pm 1.3 \\
38.7 \pm 1.8 \\
75.6 \pm 4.3 \\
2.9 \pm 1.0 \\
10.9 \pm 0.8 \\
81.2 \pm 13.5 \\
4.2 \pm 0.4 \\
0.02 \pm 0.01 \\
0.13 \pm 0.04 \\
135.7 \pm 16.3 \\
40.2 \pm 11.5 \\
2.5 \pm 0.2
\end{gathered}
$$

$21.1 \pm 1.7$

$38.0 \pm 2.3$

$71.3 \pm 3.9$

$3.0 \pm 0.2$

$9.5 \pm 0.5^{\mathrm{b}}$

$86.8 \pm 13.2$

$4.3 \pm 0.5$

$0.01 \pm 0.0$

$0.14 \pm 0.04$

$157.2 \pm 47.8$

$38.8 \pm 9.3$

$2.5 \pm 0.4$

${ }^{\text {aAt day }} 31$ of osteosarcoma progression protocol. EDTA, ethylenediaminetetraacetic acid; dDAVP, Desmopressin; RBC, red blood cells; WBC, white blood cells; AST/GOT, aspartate aminotransferase; ALT, alanine aminotransferase. ${ }^{b} \mathrm{P}<0.01$.

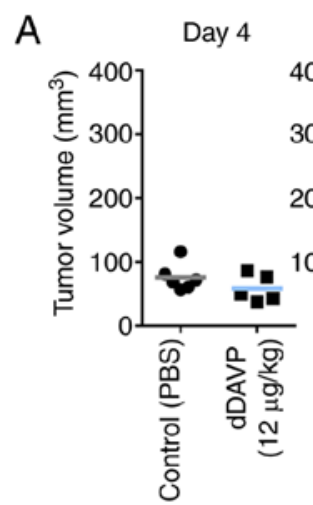

C

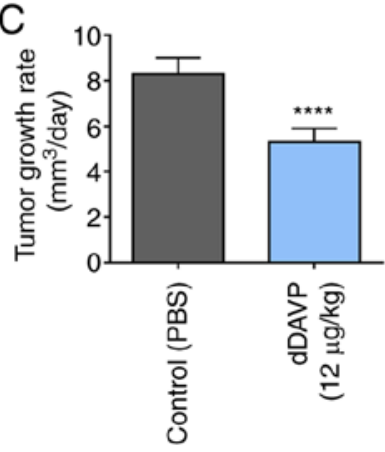

Day 15
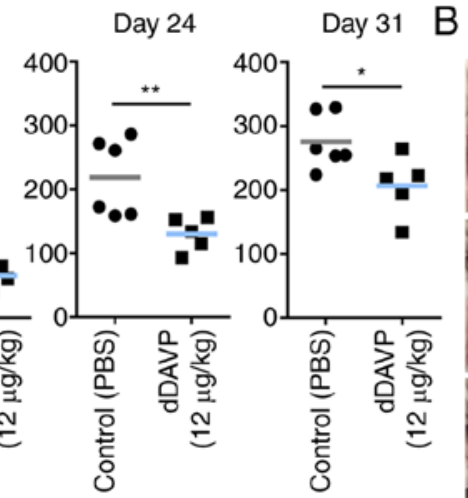

D

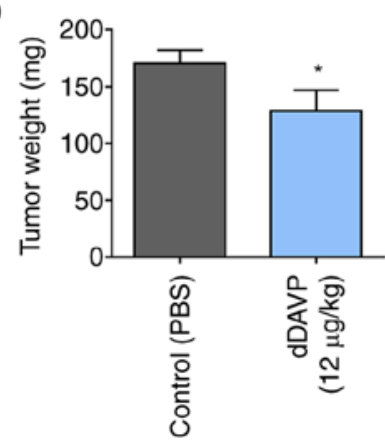

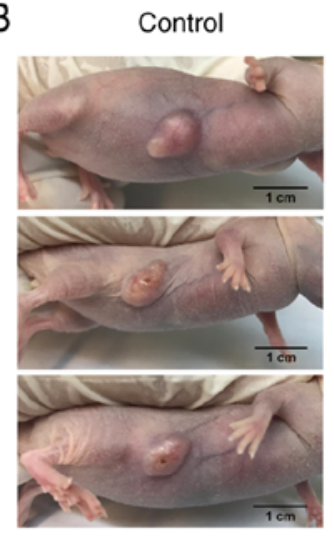

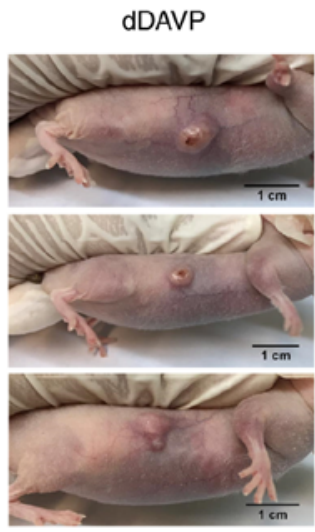

E

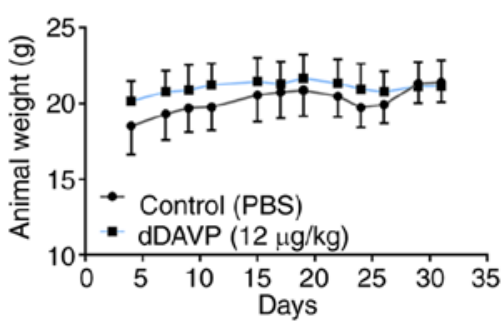

Figure 3. Sustained administration of dDAVP slows human osteosarcoma xenograft progression in vivo. (A) Tumor volume assessment over time. Animals bearing s.c MG-63 xenografts received dDAVP (12 $\mu \mathrm{g} / \mathrm{kg}$ i.v., three times a week) or PBS (control). Differences on post-engraftment days 4, 15, 24 and 31 are shown. (B) Representative images of tumor-bearing animals from different experimental groups on day 27 . Largest tumor diameter registered was 11.4 mm, and corresponded to Control group (upper photograph). Scale bar, $1 \mathrm{~cm}$. Tumor growth rates calculated between (C) days 4 and 31 , and final tumor load after xenograft resection at the (D) end of the protocol were also assessed. (E) Animal body weight was monitored three times per week. Data presented as mean \pm SEM. $n=5$ or 6 per group. ${ }^{*} \mathrm{P}<0.05,{ }^{* *} \mathrm{P}<0.01$ and ${ }^{* * * * *} \mathrm{P}<0.0001$. dDAVP, Desmopressin.

contrast to the membranous staining pattern observed in typically rounded and epithelial MCF-7 cells, AVPR2 expression pattern in spindle-shaped MDA-MB-231 cells was much more similar to that observed in MG-63 mesenchymal tumors.
AVPR2 presence was also evaluated in 6 chemotherapy-naive human conventional osteosarcoma biopsies belonging to different histological subtypes, as depicted in Fig. 5A-F. After IHC assessment, 66\% (4/6) of osteosarcoma samples displayed 

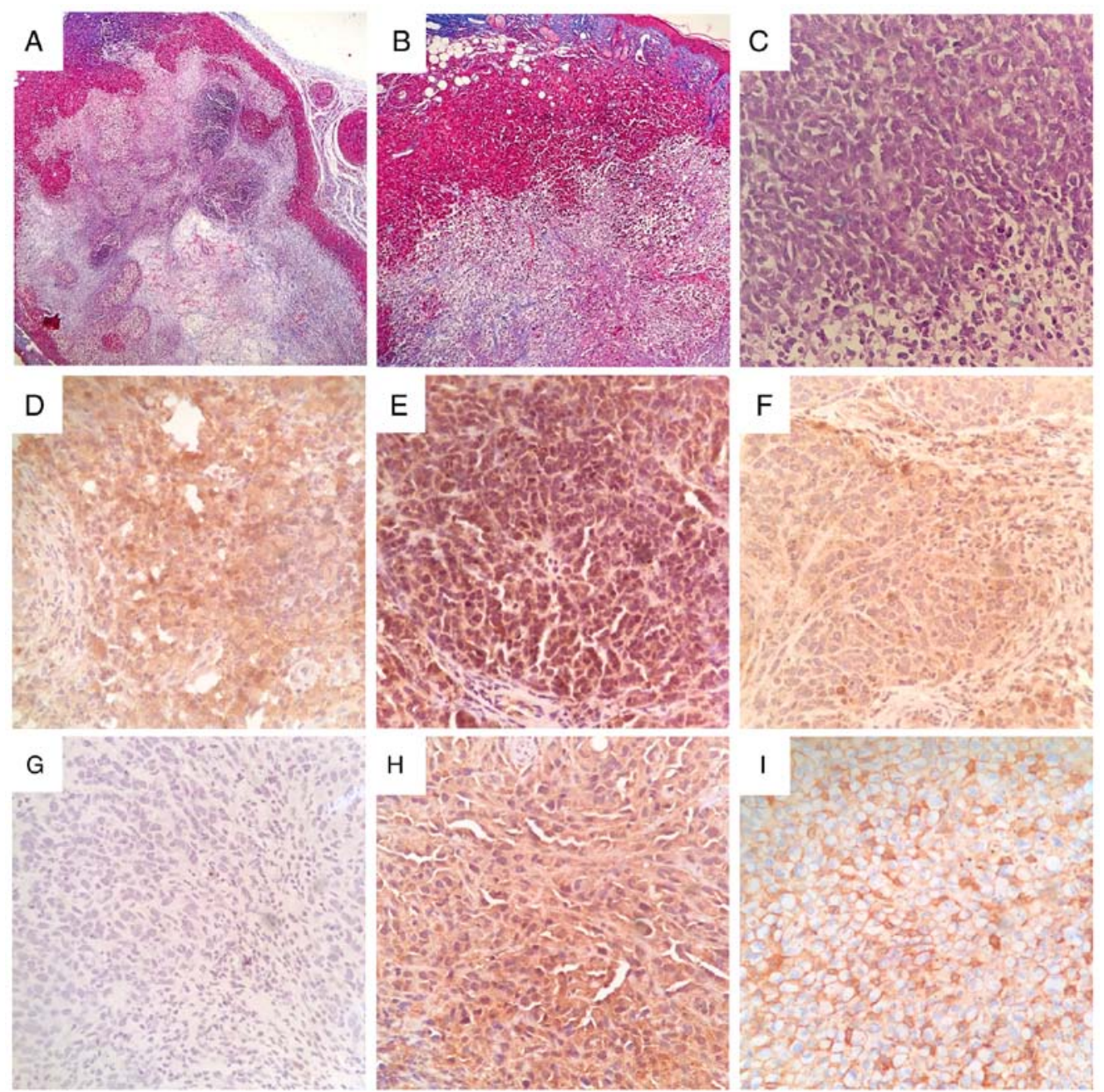

Figure 4. Histopathological features and AVPR2 expression in MG-63 osteosarcoma xenografts. Representative images of tissue sections from rapidly growing MG-63 tumors stained using Masson's trichrome staining with aniline blue at x40 and x100 magnification (A and B, respectively) or hematoxylin and eosin at x400 magnification (C) AVPR2 presence in osteosarcoma tissue was detected using IHC on formalin-fixed paraffin-embedded MG-63 xenografts using primary antibodies against human AVPR2, polymer/HRP-liked secondary antibody and hematoxylin counterstaining. (D-F) Representative microphotographs of osteosarcoma xenografts after IHC staining. Magnification, $\mathrm{x} 400$. (G) Incubation with primary antibody was omitted. Human MDA-MB-231 (H) and MCF-7 (I) xenografts were used as positive controls for AVPR2 expression. Magnification, x400. AVPR2, vasopressin membrane receptor type 2; IHC, immunohistochemistry.
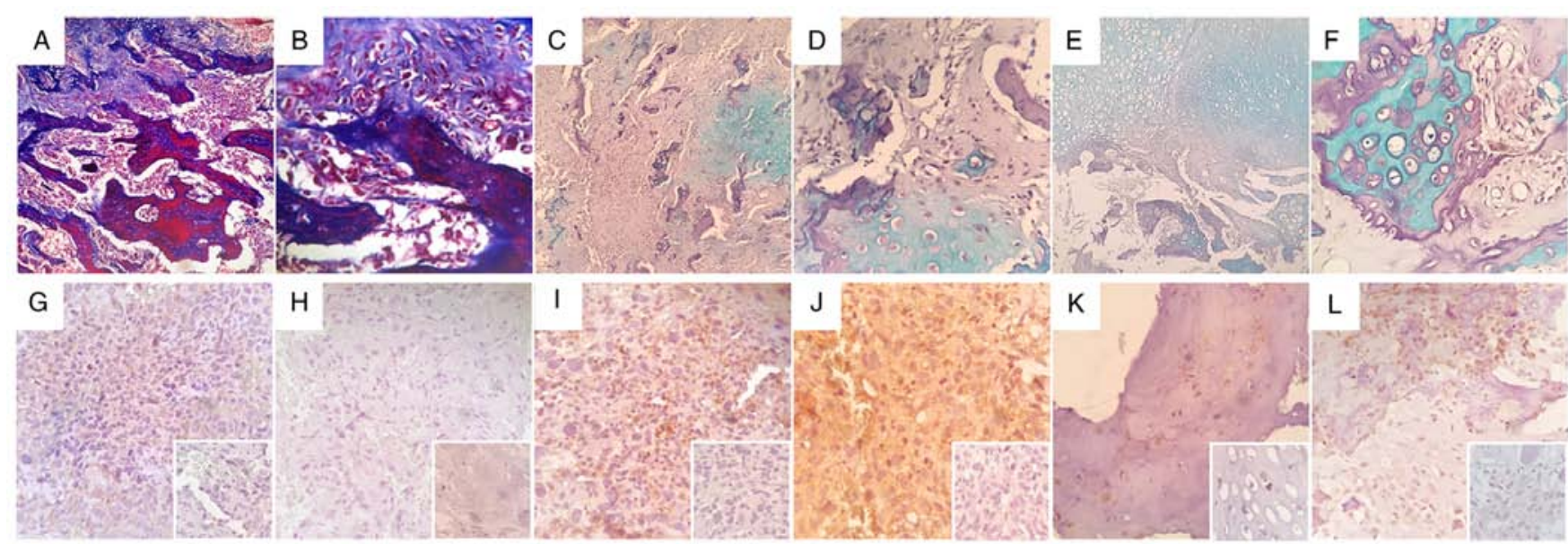

Figure 5. Immunohistochemical detection of AVPR2 in human osteosarcoma samples. Histochemical assessment revealing predominant osteoid extra-cellular matrix (A and B, x100 and x400 magnification, respectively), mixed osteoid/chondroid areas (C and D, x100 and x400 magnification, respectively) or predominant chondroid matrix (E and F, x100 and x400 magnification, respectively) in different human conventional osteosarcoma samples; (A and B) Sample \#16-10997 with Masson's trichrome staining plus aniline blue, (C and D) samples \#16-40077 and (E and F) \#16-34538 stained with hematoxylin and eosin plus alcian blue. (G-L) Representative microphotographs of human osteosarcoma samples after anti-AVPR2 immunohistochemistry detection. Human osteosarcoma samples (G) \#16-24375, (H) \#16-10997, (I) \#14-2289, (J) \#16-17130, (K) \#16-34538 and (L) \#16-40077 are presented. Inset: Incubation with primary antibody was omitted. All insets are x400 magnification. AVPR2, vasopressin membrane receptor type 2 . 
immunoreactivity, showing mild (Fig. 5K; \#16-34538), moderate (Fig. 5I and L; \#14-2289 and \#16-40077) and strong (Fig. 5J; \#16-17130) staining intensities. Osteosarcoma samples \#16-24375 and \#16-10997 (Fig. 5G and H) showed no immunoreactivity against AVPR2.

\section{Discussion}

In the present study, we report for the first time the anti-osteosarcoma activity of repurposed hemostatic drug dDAVP. Using the AVPR2-expressing MG-63 model, we showed that, in accordance with previously reported preclinical studies (14,16-18,23-25), in vitro dDAVP treatment reduced osteosarcoma cell growth and chemotaxis, and cytostatic activity was related to increased cAMP intracellular levels upon AVPR2 stimulation. Furthermore, inhibition of cAMP-degrading phosphodiesterases by addition of rolipram enhanced the antiproliferative effects of dDAVP in osteosarcoma cells. Different anticancer properties of cAMP elevating compounds, as well as cAMP synthetic derivatives, have been reported in the past, postulating the adenylate cyclase/cAMP/PKA signalling axis as a tumor suppressor system (36-39). Moreover, PDE4, a key enzyme involved in cAMP hydrolysis and degradation, is highly expressed in human mandibular osteosarcoma, and its pharmacological inhibition resulted in decreased cell survival, highlighting the role of cAMP in osteosarcoma cell proliferation (40). With the aim of gaining knowledge on AVPR2-related signaling pathways in osteosarcoma, and considering that cAMP is involved in a myriad of biological processes implicated in malignant cell growth regulation, further studies on both canonical and non-canonical AVPR2/cAMP effectors such as PKA, exchange proteins activated by cAMP 1 and 2 (Epacl and 2) and the mitogen-activated protein kinase (MAPK) on osteosarcoma cells are mandatory (41).

dDAVP antitumor activity was confirmed in vivo on human osteosarcoma xenografts growing in immunocompromised mice. Sustained intravenous administration of the compound using clinically relevant equivalent doses reduced MG-63 tumor growth without clear signs of toxicity, confirming its known profile of good tolerability and safety (11). Although growth inhibition by dDAVP in MG-63 cells was related to increased apoptosis, impaired migration and mitotic arrest, other potential and unexplored AVPR2-mediated mechanisms could be playing a role in the in vivo anti-osteosarcoma activity of the compound. It was previously reported that dDAVP is capable of blocking tumor-associated angiogenesis by favoring the production of angiostatin (ANG), an endogenous angiogenesis inhibitor specifically produced by malignant tissue. ANG overproduction resulted from breast cancer cell-mediated proteolysis of plasminogen as a result of AVPR2-mediated increased urokinase and metalloproteinase activity $(26,42)$. As a result, ANG triggers apoptosis and reduces cell growth and chemotaxis in both malignant and vascular cells $(43,44)$. Taking into account dDAVP antiangiogenic mechanisms in other tumor types as well as the key role that angiogenesis has in osteosarcoma biology, impact of dDAVP on pro- and antiangiogenic factor production by osteosarcoma cells should be assessed in the future.

As mentioned in the introduction, $20 \%$ of osteosarcoma patients present clinically detectable metastasis at diagnosis, and nearly $90 \%$ of them have lung lesions as the sole site of metastasis $(5,7)$. Interestingly, dDAVP has also proven to be a highly antimetastatic compound, being capable of impairing both experimental and spontaneous metastatic colonization of the lungs and drastically decreasing local and distant spread after surgical manipulation of aggressive tumors $(14,18,28,29)$. Besides its direct cytostatic and angiostatic effects, which depend on AVPR2 expression by tumor tissue, dDAVP antimetastatic activity was also associated to vascular release of VWF, a complex glycoprotein capable of inducing apoptosis in disseminated tumor cells (20-22). Given that metastatic osteosarcoma management remains highly challenging, further exploration of dDAVP effects on osteosarcoma dissemination and distant organ colonization is both interesting and necessary.

AVPR2 expression was confirmed in experimental osteosarcoma tumors as well as in two thirds of human osteosarcoma biopsy specimens, as evaluated by IHC. All assessed biopsies belong to untreated patients diagnosed with conventional osteosarcoma of different histological subtypes. Although the number of clinical samples was rather limited, AVPR2 expression in osteosarcoma tumors appeared to be higher than that previously reported for breast carcinoma tumors (66 vs. 33\%, respectively) (15). As new osteosarcoma cases are currently being incorporated, comparison of AVPR2 expression between primary and metastatic lesions or between pre- and post-chemotherapy, as well as correlation between AVPR2 expression and disease progression will be assessed in future studies.

Regardless of the chosen chemotherapy regimen, resection of both primary and secondary osteosarcoma remains critical for obtaining remission and improving patient survival. Osteosarcoma removal using amputation or limb salvage techniques involves complex and time-consuming surgical procedures often associated to high-volume blood loss and perioperative transfusion requirements. As observed in many types of cancer, evidence suggest a strong link between perisurgical transfusion, immunosuppression and worse prognosis in patients undergoing resection of osteosarcoma or other bone sarcomas $(45,46)$. Moreover, surgical manipulation of the tumor could favor dissemination and growth of residual cells by inducing local and systemic inflammatory responses, increased production of proangiogenic factors and suppression of antimetastatic immunity $(47,48)$. As reviewed by Pantziarka and Bouche, as a consequence of these multiple prorecurrence mechanisms associated with tumor resection, osteosarcoma is associated with alarmingly high recurrence rates in comparison to other cancer types, showing distant recurrence rates after curative intent surgery of nearly $50 \%$ (49). In this setting, perioperative administration of dDAVP during osteosarcoma removal could provide several therapeutic benefits. First, compound reduces intraoperative bleeding after releasing VWF and other hemostatic factors from vascular endothelium, reducing the need of perisurgical blood transfusion. On the other hand, dDAVP could limit osteosarcoma recurrence after surgery by impairing the survival and outgrowth of disseminated tumor cells $(50,51)$. In order to evaluate if perioperative dDAVP (administered immediately prior to surgery and during the early postoperative period) could help to antagonize deleterious mechanisms triggered by surgery, drug should be tested using experimental models of incomplete surgical resection of 
osteosarcoma tumors, especially in syngeneic and immunocompetent animal models $(52,53)$. Apart from evaluating time to local recurrence or metastatic progression to lungs, modulation of signaling pathways involved in cancer cell growth and angiogenesis should also be assessed in the postsurgical and premetastatic niches. Furthermore, given its low-associated toxicity and low production costs, dDAVP could be used as a safe and affordable perioperative therapy without increasing the risk of surgical complications or perisurgical costs.

It is important to note that osteosarcomas are known to promote a hypercoagulation state in certain patients. There is some evidence indicating that human osteosarcoma cells such as MG-63 can induce platelet aggregation activity by interacting with platelet membrane glycoproteins Ib/IX and $\mathrm{IIb} / \mathrm{III}$ a even in VWF-deficient plasma (54). However, the evidence for high VWF levels induced by dDAVP as a risk factor for venous thromboembolism is weak and perioperative use of the compound appears safe. In this regard, dDAVP administration also produces a rapid release of tissue-type plasminogen activator, with consequent activation of fibrinolysis and shortening of euglobulin lysis time. dDAVP has few hemostatic side effects but caution in small children and elderly people is recommended, due to the risk of fluid retention and hyponatremia (11).

Drug repurposing stands as an interesting approach in oncology, not only for overwhelmed health-care systems or developing countries with restrained economies, but also for rare and aggressive diseases with limited therapeutic alternatives (8-10). As observed in many diseases, prognosis of osteosarcoma patients is significantly worse in LMICs, mainly due to inadequate access to diagnostic and therapeutic resources, including surgery and radio-chemotherapy (5-7). In this setting, repurposing of blood-saving agent dDAVP for osteosarcoma treatment is highly promising due to its widely-characterized clinical use, potential antitumor activity, low treatment-associated toxicity and production costs. Compound could be administered as a co-adjuvant agent concomitantly with chemotherapy or during complex osteosarcoma removal procedures in a perioperative manner.

\section{Acknowledgements}

The authors would like to acknowledge the participation of Dr Berenice Freile (Alexander Fleming Institute, Buenos Aires, Argentina) and Dr Alejandra Scursoni (Hospital 'Dr Isidoro Iriarte', Buenos Aires, Argentina) for their involvement in histological processing and analysis, and Dr Guillermo Chantada, (Pediatric Hospital 'Prof. Dr Juan P. Garrahan', Buenos Aires, Argentina) for critical reading and reviewing of the manuscript. MG-63 and U-2 OS cell lines were kindly provided by Dr Ignacio E. León (Laboratory of Metal based Drugs, National University of La Plata, Buenos Aires, Argentina) and Dr Vanesa Gottifredi (Cell Cycle and Genomic Stability Lab, Leloir Institute Foundation, Buenos Aires, Argentina), respectively.

\section{Funding}

The current study was supported by the National Agency for the Promotion of Science and Technology (ANPCYT;
Argentina; grant no. PICT2017/2056), the National Institute of Cancer (grant no. INC2018/2020) and the National University of Quilmes Research Program grant (grant no. PUNQ1297/19).

\section{Availability of data and materials}

The datasets used and/or analyzed during the current study are available from the corresponding author on reasonable request.

\section{Authors' contributions}

NTS performed formal analysis, conducted the experiments, assessed the authenticity of raw data, contributed to the methodology and validation of the current study. LMS performed formal analysis, data curation, conducted the experiments, assessed the authenticity of raw data, contributed to the methodology of the current study and wrote the manuscript. BB contributed to the methodology and validation of the current study and reviewed and edited the manuscript. LV contributed to the methodology and validation of the current study and reviewed and edited the manuscript. GVR performed formal analysis and data curation. JG conceptualized the study, performed formal analysis, acquired funding, contributed to the methodology and validation of the current study, was involved in project administration, validation and visualization and wrote, reviewed and edited the manuscript. DFA conceptualized the study, acquired funding, was involved in project administration, reviewed and edited the manuscript and supervised the study. All authors read and approved the final manuscript.

\section{Ethics approval and consent to participate}

Clinical tumor samples were used for histological studies. Approval for the project was obtained from the Ethics Committee of the Edgardo Rebagliati Martins National Hospital. Written informed consent was obtained from all patients or their parents prior to the samples' examination. All animal protocols were carried out in accordance with the Guide for the Care and Use of Laboratory Animals as adopted by the U.S. National Institutes of Health (NIH Publications No. 8023, revised 1978) and were approved by our institutional Animal Care Committee UNQ-CICUAL (Resolution CD CyT No. 075/14); Protocol code: 011-15. Results were reported following the ARRIVE guidelines.

\section{Patient consent for publication}

Not applicable.

\section{Competing interests}

The authors declare that they have no competing interests.

\section{References}

1. Mirabello L, Troisi RJ and Savage SA: International osteosarcoma incidence patterns in children and adolescents, middle ages and elderly persons. Int J Cancer 125: 229-234, 2009.

2. Durfee RA, Mohammed M and Luu HH: Review of osteosarcoma and current management. Rheumatol Ther 3: 221-243, 2016. 
3. Marec-Berard P, Laurence V, Occean BV, Ray-Coquard I, Linassier C, Corradini N, Collard O, Chaigneau L, Cupissol D, Kerbrat $\mathrm{P}$, et al: Methotrexate-Etoposide-ifosfamide compared with doxorubicin-cisplatin-ifosfamide chemotherapy in osteosarcoma treatment, patients aged 18-25 years. J Adolesc Young Adult Oncol 9: 172-182, 2020.

4. Meazza C and Scanagatta P: Metastatic osteosarcoma: A challenging multidisciplinary treatment. Expert Rev Anticancer Ther 16: 543-556, 2016

5. Moreno F, Cacciavillano W, Cipolla M, Coirini M, Streitenberger P, Lopez Marti J, Palladino M, Morici M, Onoratelli M, Drago G, et al: Childhood osteosarcoma: Incidence and survival in argentina. report from the national pediatric cancer registry, ROHA network 2000-2013. Pediatr Blood Cancer 642017.

6. Revon-Riviere G, Banavali S, Heississen L, Gomez Garcia W, Abdolkarimi B, Vaithilingum M, Li CK, Leung PC, Malik P, Pasquier E, et al: Metronomic chemotherapy for children in low- and middle-income countries: Survey of current practices and opinions of pediatric oncologists. J Glob Oncol 5: 1-8, 2019.

7. Gyawali B, Bouche G, Crisp N and André N: Challenges and opportunities for cancer clinical trials in low- and middle-income countries. Nature Cancer 1: 142-145, 2020.

8. Bertolini F, Sukhatme VP and Bouche G: Drug repurposing in oncology-patient and health systems opportunities. Nat Rev Clin Oncol 12: 732-742, 2015.

9. Pushpakom S, Iorio F, Eyers PA, Escott KJ, Hopper S, Wells A, Doig A, Guilliams T, Latimer J, McNamee C, et al: Drug repurposing: Progress, challenges and recommendations. Nat Rev Drug Discov 18: 41-58, 2019.

10. Pantziarka $P$ and Andre N: Editorial: Drug repurposing. Front Med (Lausanne) 6: 154, 2019.

11. Svensson PJ, Bergqvist PB, Juul KV and Berntorp E: Desmopressin in treatment of haematological disorders and in prevention of surgical bleeding. Blood Rev 28: 95-102, 2014

12. Kaufmann JE, Oksche A, Wollheim CB, Gunther G, Rosenthal W and Vischer UM: Vasopressin-induced von Willebrand factor secretion from endothelial cells involves V2 receptors and cAMP. J Clin Invest 106: 107-116, 2000.

13. Garona J, Pifano M, Pastrian MB, Gomez DE, Ripoll GV and Alonso DF: Addition of vasopressin synthetic analogue [V(4) Q(5)]dDAVP to standard chemotherapy enhances tumour growth inhibition and impairs metastatic spread in aggressive breast tumour models. Clin Exp Metastasis 33: 589-600, 2016.

14. Garona J, Pifano M, Scursoni AM, Gomez DE, Alonso DF and Ripoll GV: Insight into the effect of the vasopressin analog desmopressin on lung colonization by mammary carcinoma cells in BALB/c mice. Anticancer Res 34: 4761-4765, 2014.

15. Weinberg RS, Grecco MO, Ferro GS, Seigelshifer DJ, Perroni NV, Terrier FJ, Sánchez-Luceros A, Maronna E, Sánchez-Marull R, Frahm I, et al: A phase II dose-escalation trial of perioperative desmopressin (1-desamino-8-d-arginine vasopressin) in breast cancer patients. Springerplus 4: 428, 2015.

16. Pifano M, Garona J, Capobianco CS, Gonzalez N, Alonso DF and Ripoll GV: Peptide agonists of vasopressin V2 receptor reduce expression of neuroendocrine markers and tumor growth in human lung and prostate tumor cells. Front Oncol 7: $11,2017$.

17. Pifano M, Garona J, Sobol NT, Alberto M, Alonso DF and Ripoll GV: Search of vasopressin analogs with antiproliferative activity on small-cell lung cancer: Drug design based on two different approaches. Future Med Chem 10: 879-894, 2018.

18. Ripoll GV, Garona J, Hermo GA, Gomez DE and Alonso DF: Effects of the synthetic vasopressin analog desmopressin in a mouse model of colon cancer. Anticancer Res 30: 5049-5054, 2010.

19. Garona J, Sobol NT, Pifano M, Segatori VI, Gomez DE, Ripoll GV and Alonso DF: Preclinical efficacy of [V4 Q5] dDAVP, a second generation vasopressin analog, on metastatic spread and tumor-associated angiogenesis in colorectal cancer. Cancer Res Treat 51: 438-450, 2019.

20. Terraube V, Pendu R, Baruch D, Gebbink MF, Meyer D, Lenting PJ and Denis CV: Increased metastatic potential of tumor cells in von Willebrand factor-deficient mice. J Thromb Haemost 4: 519-526, 2006

21. Mochizuki S, Soejima K, Shimoda M, Abe H, Sasaki A, Okano HJ, Okano H and Okada Y: Effect of ADAM28 on carcinoma cell metastasis by cleavage of von Willebrand factor. J Natl Cancer Inst 104: 906-922, 2012
22. Gragnano F, Sperlongano S, Golia E, Natale F, Bianchi R, Crisci M, Fimiani F, Pariggiano I, Diana V, Carbone A, et al: The role of von willebrand factor in vascular inflammation: From pathogenesis to targeted therapy. Mediators Inflamm 2017: 5620314, 2017.

23. Keegan BP, Akerman BL, Pequeux $\mathrm{C}$ and North WG: Provasopressin expression by breast cancer cells: Implications for growth and novel treatment strategies. Breast Cancer Res Treat 95: 265-277, 2006

24. Garona J, Pifano M, Orlando UD, Pastrian MB, Iannucci NB, Ortega HH, Podesta EJ, Gomez DE, Ripoll GV and Alonso DF: The novel desmopressin analogue [V4Q5]dDAVP inhibits angiogenesis, tumour growth and metastases in vasopressin type 2 receptor-expressing breast cancer models. Int J Oncol 46 : 2335-2345, 2015.

25. Sasaki H, Klotz LH, Sugar LM, Kiss A and Venkateswaran V A combination of desmopressin and docetaxel inhibit cell proliferation and invasion mediated by urokinase-type plasminogen activator (uPA) in human prostate cancer cells. Biochem Biophys Res Commun 464: 848-854, 2015.

26. Ripoll GV, Garona J, Pifano M, Farina HG, Gomez DE and Alonso DF: Reduction of tumor angiogenesis induced by desmopressin in a breast cancer model. Breast Cancer Res Treat 142: 9-18, 2013.

27. Ripoll GV, Farina HG, Yoshiji H, Gomez DE and Alonso DF: Desmopressin reduces melanoma lung metastasis in transgenic mice overexpressing tissue inhibitor of metalloproteinases-1. In Vivo 20: 881-885, 2006.

28. Alonso DF, Skilton G, Farias EF, Bal de Kier Joffe E and Gomez DE: Antimetastatic effect of desmopressin in a mouse mammary tumor model. Breast Cancer Res Treat 57: 271-275, 1999.

29. Giron S, Tejera AM, Ripoll GV, Gomez DE and Alonso DF Desmopressin inhibits lung and lymph node metastasis in a mouse mammary carcinoma model of surgical manipulation. J Surg Oncol 81: 38-44, 2002.

30. Hermo GA, Torres P, Ripoll GV, Scursoni AM, Gomez DE, Alonso DF and Gobello C: Perioperative desmopressin prolongs survival in surgically treated bitches with mammary gland tumours: A pilot study. Vet J 178: 103-108, 2008

31. Hermo GA, Turic E, Angelico D, Scursoni AM, Gomez DE, Gobello C and Alonso DF: Effect of adjuvant perioperative desmopressin in locally advanced canine mammary carcinoma and its relation to histologic grade. J Am Anim Hosp Assoc 47: 21-27, 2011

32. Iseas S, Roca EL, O'Connor JM, Eleta M, Sanchez-Luceros A, Di Leo D, Tinelli M, Fara ML, Spitzer E, Demarco IA, et al: Administration of the vasopressin analog desmopressin for the management of bleeding in rectal cancer patients: Results of a phase I/II trial. Invest New Drugs 38: 1580-1587, 2020.

33. Nair $A B$ and Jacob $S$ : A simple practice guide for dose conversion between animals and human. J Basic Clin Pharm 7: 27-31, 2016.

34. Ray G, Banerjee S, Saxena NK, Campbell DR, Van Veldhuizen P and Banerjee SK: Stimulation of MCF-7 tumor progression in athymic nude mice by 17 beta-estradiol induces WISP-2/CCN5 expression in xenografts: A novel signaling molecule in hormonal carcinogenesis. Oncol Rep 13: 445-448, 2005.

35. Pastrian MB, Guzman F, Garona J, Pifano M, Ripoll GV, Cascone O, Ciccia GN, Albericio F, Gómez DE, Alonso DF and Iannucci NB: Structure-activity relationship of 1-desamino-8-D-arginine vasopressin as an antiproliferative agent on human vasopressin V2 receptor-expressing cancer cells. Mol Med Rep 9: 2568-2572, 2014.

36. Carie AE and Sebti SM: A chemical biology approach identifies a beta-2 adrenergic receptor agonist that causes human tumor regression by blocking the Raf-1/Mek-1/Erk1/2 pathway. Oncogene 26: 3777-3788, 2007.

37. Insel PA, Zhang L, Murray F, Yokouchi $\mathrm{H}$ and Zambon AC: Cyclic AMP is both a pro-apoptotic and anti-apoptotic second messenger. Acta Physiol (Oxf) 204: 277-287, 2012.

38. Naviglio S, Di Gesto D, Romano M, Sorrentino A, Illiano F, Sorvillo L, Abbruzzese A, Marra M, Caraglia M, Chiosi E, et al: Leptin enhances growth inhibition by cAMP elevating agents through apoptosis of MDA-MB-231 breast cancer cells. Cancer Biol Ther 8: 1183-1190, 2009.

39. Castoria G, Migliaccio A, D'Amato L, Di Stasio R, Ciociola A, Lombardi M, Bilancio A, Di Domenico M, de Falco A and Auricchio F: Integrating signals between cAMP and MAPK pathways in breast cancer. Front Biosci 13: 1318-1327, 2008. 
40. Narita M, Murata T, Shimizu K, Sugiyama T, Nakagawa T, Manganiello VC and Tagawa T: Phosphodiesterase 4 in osteoblastic osteosarcoma cells as a potential target for growth inhibition. Anticancer Drugs 14: 377-381, 2003.

41. Fajardo AM, Piazza GA and Tinsley HN: The role of cyclic nucleotide signaling pathways in cancer: Targets for prevention and treatment. Cancers (Basel) 6: 436-458, 2014.

42. Ripoll G, Iannucci N, Giron S, Cascone O, Gomez D and Alonso D: Angiostatic activity of 1-Deamino-8-D-Arginine vasopressin and novel peptide analogues in breast cancer cells. Cancer Res 68: 295, 2008.

43. Sakurai T and Kudo M: Signaling pathways governing tumor angiogenesis. Oncology 81 (Suppl 1): S24-S29, 2011.

44. Westphal JR, Van't Hullenaar R, Geurts-Moespot A, Sweep FC, Verheijen JH, Bussemakers MM, Askaa J, Clemmensen I, Eggermont AA, Ruiter DJ and De Waal RM: Angiostatin generation by human tumor cell lines: Involvement of plasminogen activators. Int J Cancer 86: 760-767, 2000.

45. Chesi R, Cazzola A, Bacci G, Borghi B, Balladelli A and Urso G: Effect of perioperative transfusions on survival in osteosarcoma treated by multimodal therapy. Cancer 64: 1727-1737, 1989.

46. Park JH, Hong SH and Jang WY: Restrictive allogeneic blood transfusion strategy in patients with extremity bone sarcomas. Medicine (Baltimore) 98: e18482, 2019.

47. Horowitz M, Neeman E, Sharon E and Ben-Eliyahu S: Exploiting the critical perioperative period to improve long-term cancer outcomes. Nat Rev Clin Oncol 12: 213-226, 2015.

48. Garona J and Alonso DF: Perioperative biology in primary breast cancer: Selective targeting of vasopressin type 2 receptor using desmopressin as a novel therapeutic approach. Breast Cancer Res Treat 158: 597-599, 2016
49. Pantziarka $P$ and Bouche G: Perioperative therapies-using repurposed drugs to improve cancer surgery outcomes. Cancer J 25: $100-105,2019$

50. Garona J, Sobol NT and Alonso DF: Impact of Perioperative blood transfusion on survival among women with breast cancer: Potential benefits of blood-saving agent desmopressin use during surgery. Am J Ther 25: e569-e570, 2018.

51. Garona J, Sobol NT, Solerno LM and Alonso DF: Potential use of desmopressin during hepatic resection for colorectal liver metastases. J Surg Res 237: 1-2, 2019.

52. Gottardo MF, Capobianco CS, Sidabra JE, Garona J, Perera Y, Perea SE, Alonso DF and Farina HG: Preclinical efficacy of CIGB-300, an anti-CK2 peptide, on breast cancer metastasic colonization. Sci Rep 10: 14689, 2020.

53. Guijarro MV, Ghivizzani SC and Gibbs CP: Animal models in osteosarcoma. Front Oncol 4: 189, 2014

54. Clezardin P, Drouin J, Morel-Kopp MC, Hanss M, Kehrel B, Serre CM, Kaplan C and Delmas PD: Role of platelet membrane glycoproteins Ib/IX and IIb/IIIa, and of platelet alpha-granule proteins in platelet aggregation induced by human osteosarcoma cells. Cancer Res 53: 4695-4700, 1993.

(i) $(-)$ This work is licensed under a Creative Commons Attribution-NonCommercial-NoDerivatives 4.0 International (CC BY-NC-ND 4.0) License. 